\title{
Los microorganismos que habitan los bañados de desborde fluvial como indicadores de los efectos de la urbanización y la actividad agropecuaria
}

María Fernanda Alvarez ${ }^{1}$, Hernán Hugo Benítez ${ }^{1}$, Javier Ricardo Garcia de Souza', Delia Elena Bauer ${ }^{1}$, Alan Santiago Tarda ${ }^{1}$, María Mercedes Nicolosi Gelis ${ }^{1}$, Analía Díaz ${ }^{1}$, Mario Carlos Nazareno Saparrat ${ }^{2,3,4}$ y Nora Gómez ${ }^{1}$.

${ }^{1}$ Instituto de Limnología “Dr. Raúl A. Ringuelet" (CONICET-UNLP), La Plata, Argentina. ${ }^{2}$ Instituto de Fisiología Vegetal (INFIVE), Consejo Nacional de Investigaciones Científicas y técnicas (CONICET), Universidad Nacional de La Plata CCT-La Plata, Argentina.

${ }^{3}$ Instituto de Botánica Carlos Spegazzini, Facultad de Ciencias Naturales y Museo, Universidad Nacional de La Plata, Argentina.

${ }^{4}$ Cátedra de Microbiología Agrícola, Departamento de Ciencias Biológicas, Facultad de Ciencias Agrarias y Forestales, Universidad Nacional de La Plata, Argentina.

E-mail: feralvarez@ilpla.edu.ar

RESUMEN. Los bañados de desborde fluvial (BDF) desempeñan un papel importante en la ecología de las cuencas fluviales y en su capacidad de autodepuración. La demanda antrópica de espacio y agua pone en riesgo su biota y los beneficios ecosistémicos que brindan. Los objetivos del trabajo fueron analizar los ensambles de microorganismos que habitan en BDF de los partidos de La Plata y de Magdalena (Buenos Aires), relacionar sus variaciones con el impacto antrópico y evaluar cómo la microbiota puede ser indicadora de estos cambios ambientales. Se estudiaron los ensambles de diatomeas bentónicas, fitoplancton, zooplancton, ostrácodos y hongos de cuatro BDF: dos de ubicación periurbana con uso del suelo agrícola y hortícola (Del Gato y Carnaval) y dos de ubicación rural y uso ganadero (Cajaravillas y Chubichaminí). Las diferencias vinculadas con el uso del suelo se expresaron en la mayoría de los grupos, y los BDF se agruparon en rurales (con más especies sensibles a la contaminación y a la eutrofización) y periurbanos (donde fueron más importantes las especies muy tolerantes). En estos últimos, tanto los grandes grupos taxonómicos como los niveles tróficos estuvieron más relacionados con la vía detritívora que con la fotosintética, y en esos ambientes también fueron más relevantes las estrategias de los organismos para afrontar el estrés hídrico. Los resultados evidencian los efectos de la presión humana, la importancia que adquieren los microorganismos para evaluarla y la necesidad de una planificación adecuada del uso del territorio para evitar la pérdida de funciones y beneficios en estos ambientes.

Palabras clave: Cambios ambientales, humedales, microbiota acuática, servicios ecosistémicos, uso del suelo.

ABSTRACT. Riverine wetlands (RW) play a key role in the ecology of river basins and their selfpurification capacity. The anthropic demand for space and water puts their biota and the ecosystem benefits they provide at risk. The objectives of this work were to analyze the assemblages of microorganisms that inhabit the RW of La Plata and Magdalena (Buenos Aires), relate their variations to anthropogenic impact and evaluate how the microbiota can be an indicator of these environmental changes. Benthic diatoms, phytoplankton, zooplankton, ostracods, and fungi assemblages of four RW were studied: two of peri-urban location with agricultural and horticultural land use (Del Gato and Carnaval), and two of rural location and livestock land use (Cajaravillas and Chubichaminí). Differences linked to land uses were clearly expressed in most groups, with RWs grouped into rural (with more species sensitive to pollution and eutrophication) and peri-urban (with more species highly tolerant). In the latter, both the main taxonomic groups and the trophic 
levels were more restricted and more closely related to the detritivorous pathway than to the photosynthetic one, and the strategies to face water stress were emphasized. The results show the effects of human pressure, the importance that microorganisms acquire in its evaluation and the need of an adequate use of the territory to avoid the loss of functions and benefits of these environments.

Keywords: Aquatic microbiota, ecosystem services, environmental changes, land use, wetlands.

\section{INTRODUCCIÓN}

Los humedales desempeñan un papel importante en la ecología de las cuencas fluviales (EPA, 2015). Su función biológica, aunque obvia para la mayoría de los biólogos o ecólogos, a menudo es cuestionada por otros sectores, especialmente cuando los humedales son de pequeño tamaño, transitorios o temporales y obstaculizan los intentos de cultivar o de construir caminos y casas (Dodson \& Lillie, 2001). Estos ambientes brindan numerosos servicios ecosistémicos (Davidson, 2014); incluso los pequeños pueden regular los flujos en períodos lluviosos, proporcionar humedad para el desarrollo de la biota en períodos secos o eliminar y retener sedimentos, nutrientes y contaminantes purificando el agua (Davidson, 2014; Millennium Ecosystem Assessment, 2005; Strayer \& Dudgeon, 2010). La combinación de aguas poco profundas y altos niveles de nutrientes es ideal para el desarrollo de los organismos, por lo que estos ambientes albergan gran diversidad (Dodson \& Lillie, 2001), ofreciendo sitios de cría, refugio y alimentación para numerosas especies (Davidson, 2014; Millennium Ecosystem Assessment, 2005; Staryer \& Dudgeon, 2010).

La progresiva pérdida y destrucción de los humedales efímeros o intermitentes (aproximadamente el $87 \%$ ha desaparecido desde principios del siglo XVIII; Davidson, 2014) junto con la contaminación, es uno de los mayores riesgos que afrontan los organismos que viven en estos ambientes (Davidson, 2014; EPA, 2015). Muchas veces la erosión, el aumento de la turbidez, la regulación del flujo de agua, la introducción de especies exóticas, la contaminación por pesticidas, fertilizantes $\mathrm{u}$ otros productos que afectan la calidad de agua (Davidson, 2014; Dodson et al., 2000; Dodson \& Lillie, 2001; Millennium Ecosystem
Assessment 2005; Staryer \& Dudgeon, 2010) son factores que determinan qué organismos se pueden encontrar, cuántos e incluso de qué tamaños. En estos ambientes, las microalgas junto con las macrófitas son las principales productoras fotosintéticas de carbono orgánico y proporcionan energía para niveles tróficos superiores. El zooplancton también juega un papel crucial (Bruce et al., 2006; Jeppesen et al. 1991) ya que cumple funciones integradoras, al participar en la transferencia de materia, energía y en la autorregulación de los cuerpos de agua (Jeppesen et al., 2011; Tudor et al., 2015). A su vez, cuando los organismos mueren, otros son capaces de descomponerlos recirculando la materia como diferentes fracciones de carbono en el bucle microbiano (Meyer, 1994).

Los humedales ribereños actúan también como bancos de semillas e inóculos de los organismos, los cuales pueden sobrevivir durante largos períodos de tiempo (Hairston, 1996), y dispersarse entre los hábitats en las planicies de inundación de ríos o arroyos una vez que se restablecen las conexiones por los eventos de lluvia (Medley \& Havel, 2007; Zaplara et al., 2018). En la llanura pampeana es frecuente reconocer, a lo largo de los cursos de agua, humedales ribereños que se denominan bañados de desborde fluvial (BDF). Estos ambientes se alimentan por desborde de ríos y arroyos y por aporte de agua subterránea. Dependen de pequeñas inundaciones periódicas para reciclar y redistribuir nutrientes y organismos (Brinson et al., 1995; Ringuelet, 1962), y aumentar la diversidad de las comunidades (Bayley, 1995). La presencia de estos ambientes en las cuencas resulta importante para su funcionamiento y estructura; sin embargo, muchos de ellos están sufriendo los efectos de las transformaciones en los usos del suelo, conduciéndolos a un progresivo deterioro ambiental y en algunos 
casos hasta la extinción por el avance de las actividades antrópicas (López, 2018). Esto ocasiona pérdidas de superficies valiosas de las cuencas y con ellas se reducen los beneficios ecosistémicos que estos sistemas brindan. Por estas razones, el objetivo del presente estudio fue analizar de manera cuali-cuantitativa los ensambles de microorganismos que habitan estos humedales (fitoplancton, zooplancton, diatomeas bentónicas, hongos acuáticos y ostrácodos), vinculando lo observado con cambios ambientales; determinar el papel de esta microbiota en la estructura y funcionamiento de los BDF y cómo puede ser utilizada como indicadora del estrés al que están expuestos debido a las actividades antrópicas.

\section{MATERIALES Y MÉTODOS}

\section{Sitios de estudio}

Se estudiaron cuatro BDF ubicados en los arroyos Carnaval, Del Gato, Cajaravillas y Chubichaminí que atraviesan áreas periurbanas influenciadas por la actividad agrícola y la urbanización, ubicados en los Partidos de La Plata y de Magdalena (Figura 1). Desde principios del siglo XX estos ambientes soportan constantes transformaciones en los usos del suelo de sus cuencas, con un crecimiento exponencial del área afectada por invernáculos a partir de la década de 1990 (García, 2011). Los BDF localizados en los arroyos Carnaval y Del Gato, tienen una superficie de 0,02 y 0,08 $\mathrm{km}^{2}$ respectivamente y una profundidad que no supera los 0,6 m; en tanto que los situados en los arroyos Chubichaminí y Cajaravillas, tienen una superficie de 0,09 y 0,11 $\mathrm{km}^{2}$ respectivamente y alcanzan hasta $1 \mathrm{~m}$ de profundidad. Los BDF del Carnaval y Del Gato se localizan en una zona periurbana, el primero rodeado por cultivos de soja y el segundo influenciado por la actividad hortícola. La excesiva extracción de agua para riego de cultivos y para el abastecimiento de la ciudad de La Plata y sus alrededores ha provocado el descenso local de los niveles freáticos, particularmente en el sector sur de la ciudad. Esta situación ha sido señalada por Rodrigues Capítulo et al. (2020) quienes la indican como la causa de la pérdida del aporte de agua subterránea al caudal base de los arroyos en donde se encuentran estos humedales. Los BDF localizados en áreas rurales (Chubichaminí y Cajaravillas), a diferencia de los anteriores, reciben aportes de agua subterránea y superficial, siendo el primero de mayor importancia cuantitativa. El uso del suelo en los alrededores de ambos es la ganadería extensiva. De acuerdo con estudios recientes, los BDF ubicados en el área periurbana con

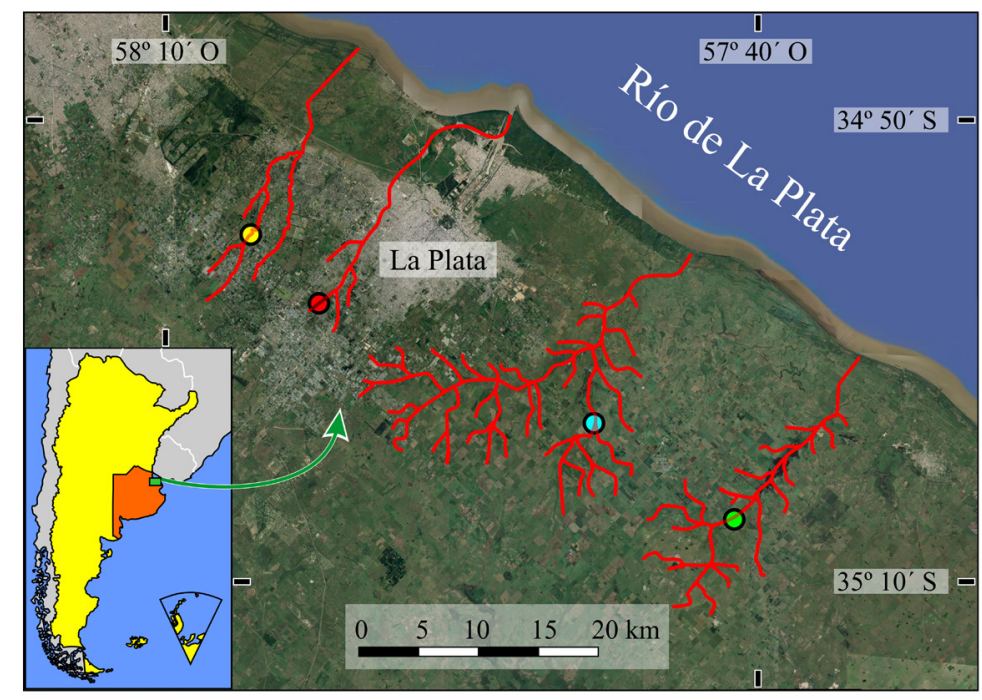

Figura 1. Mapa del área de estudio con los cuatro bañados de desborde fluvial (BDF) estudiados: O ,Carnaval; O,Del Gato; O,Cajaravillas; O,Chubichaminí.

Figure 1. Study area with the four BDFs studied:O,Carnaval; O, Del Gato; O, Cajaravillas; O,Chubichaminí. 
actividad agrícola exhibieron concentraciones significativamente mayores de fósforo y nitrógeno totales que los ubicados en áreas rurales con ganadería extensiva mientras que en éstos se observaron valores más elevados de conductividad y DQO (Tabla 1). Además, los BDF ubicados en áreas rurales tuvieron una elevada carga bacteriana de origen fecal proveniente del ganado expresada por Escherichia coli (Cochero et al., 2020; Tabla 1), en tanto que existen registros de mayor concentración de agroquímicos en los bañados periurbanos, que en otros bañados vecinos a los de ubicación rural y sujetos al mismo uso del suelo (Mac Loughlin et al., 2017; Rimoldi et al., 2018, Solís et al., 2018).

\section{Comunidades}

Para el análisis de las comunidades de productores primarios (diatomeas bentónicas y fitoplancton) y de consumidores (zooplancton), se tomaron muestras por triplicado en cada uno de los BDF en otoño, invierno y primavera de 2017 y verano de 2018. Las muestras para analizar los ensambles de ostrácodos fueron colectadas durante primavera y verano (2017, 2018); en tanto que para los descomponedores (hongos acuáticos) los muestreos se realizaron durante verano, otoño e invierno de 2018.

Durante el verano de 2018, en concordancia con un período climático de "La Niña", se produjo una sequía que ocasionó que los BDF del arroyo Carnaval y Del Gato permanecieran secos, por lo cual no se pudieron extraer las muestras biológicas durante este período.

\section{Productores primarios}

Para la extracción de las diatomeas bentónicas se colectaron muestras compuestas por 5 submuestras de los primeros $5-10 \mathrm{~mm}$ de la capa superficial del sedimento del lecho de los BDF. A tal fin se empleó una pipeta con un aspirador en su extremo (Gómez et al., 2009; Licursi \& Gómez, 2002) con la que se succionaron $4 \mathrm{~mL}$ de biofilm por submuestra. Las muestras se fijaron con formol $4 \%$ para su preservación. Las diatomeas fueron separadas del sedimento por medio de Ludox (de Jonge, 1979) y fueron oxidadas con $\mathrm{H}_{2} \mathrm{O}_{2}$ (100 volúmenes). Luego fueron montadas en portaobjetos utilizando Naphrax ${ }^{\circledR}$ (Stevenson \& Bahls, 1999). Se identificaron un total de 400 valvas por preparado utilizando un microscopio Olympus BX 51 con contraste de fases e interferencial a una magnificación de 1000X, con el fin de obtener la abundancia relativa de cada taxón (Descy \& Coste, 1991). La identificación de las especies se realizó principalmente según: Krammer \& Lange-Bertalot (1986, 1988, 1991a, 1991 b); Lange-Bertalot (2000); Metzeltin \&

Tabla 1. Parámetros físicos - químicos y densidad Escherichia coli (valores promedios \pm desvío estándar) de los BDF Chubichaminí, Cajaravillas, Del Gato y Carnaval, correspondientes a los muestreos realizados durante 2017-2018 de extraídos de Rodrigues Capítulo et al. (2020) y Cochero et al. (2020).

Table 1. Physical-chemical parameters and density of Escherichia coli (average values \pm standard deviation) of the Chubichaminí, Cajaravillas, Del Gato and Carnaval BDFs, corresponding to the samplings carried out during 2017-2018, extracted from Rodrigues Capítulo et al. (2020) and Cochero et al. (2020).

\begin{tabular}{lcccc}
\hline & Chubichaminí & Cajaravillas & Del Gato & Carnaval \\
\hline Temperatura $\left({ }^{\circ} \mathrm{C}\right)$ & $19,8( \pm 6,6)$ & $21,5( \pm 7,0)$ & $17,1( \pm 6,4)$ & $15,4( \pm 6,8)$ \\
$\mathrm{pH}$ & $8,1( \pm 0,4)$ & $8,1( \pm 0,3)$ & $8,0( \pm 0,3)$ & $8,0( \pm 0,2)$ \\
Conductivdad $(\mu \mathrm{S} / \mathrm{cm})$ & $918( \pm 150)$ & $855( \pm 38)$ & $614( \pm 108)$ & $769( \pm 22)$ \\
\% de saturación de oxigeno & $74( \pm 20)$ & $62( \pm 21)$ & $49( \pm 24)$ & $71( \pm 12)$ \\
Turbidez (UNT) & $277( \pm 240)$ & $73( \pm 62)$ & $59( \pm 39)$ & $106( \pm 61)$ \\
Nitrógeno Total (mg/L) & $2,81( \pm 0,89)$ & $2,59( \pm 0,79)$ & $8,40( \pm 7,23)$ & $6,45( \pm 2,15)$ \\
Fósforo total (mg/L) & $0,38( \pm 0,12)$ & $0,52( \pm 0,24)$ & $1,09( \pm 0,35)$ & $1,25( \pm 0,31)$ \\
DQO (mg/L) & $71( \pm 26)$ & $36( \pm 17)$ & $24( \pm 8)$ & $24( \pm 13)$ \\
DBO $5(\mathrm{mg} / \mathrm{L})$ & $11( \pm 4)$ & $10( \pm 3)$ & $8( \pm 3)$ & $8( \pm 5)$ \\
Escherichia coli $(\mathrm{UFC} / \mathrm{mL})$ & $67( \pm 72)$ & $46( \pm 56)$ & $21( \pm 29)$ & $9( \pm 12)$ \\
& & & & \\
\hline
\end{tabular}


Lange-Bertalot (1998, 2005, 2007). Las especies fueron clasificadas según su tolerancia a la eutrofización y contaminación por materia orgánica (Gómez \& Licursi, 2001) en 3 categorías: "Muy tolerantes", "Tolerantes" y "Sensibles". Para el análisis del fitoplancton se colectaron muestras de agua subsuperficial $(125 \mathrm{~mL})$, que fueron fijadas in situ con formol al $2 \%$. Para la determinación taxonómica se utilizó un microscopio óptico Olympus BX 50 con contraste de fases e interferencial a 1000X. La cuantificación se realizó de acuerdo con Utermöhl (1958) y Lund et al. (1958) utilizando un microscopio invertido Olympus CK2 con una magnificación de 400X. Las muestras se dejaron sedimentar luego del agregado de lugol acético (Prygiel \& Leitao, 1994). Con la densidad obtenida en los recuentos se calculó la abundancia relativa de las especies. Los taxa se clasificaron en grandes grupos taxonómicos y también considerando su hábito de vida (euplanctónicas -organismos estrictamente planctónicos- o ticoplanctónicas -organismos que proceden de otras comunidades y que ocasionalmente pueden formar parte de la comunidad planctónica) y su tolerancia a la contaminación con materia orgánica y a la eutrofización (sensibles, tolerantes y muy tolerantes). La identificación de las especies se llevó a cabo principalmente siguiendo a Desikachary (1950), Komárek \& Fott (1983), Tell \& Conforti (1986), Komárek \& Anagnostidis (2005) y bibliografía para diatomeas detallada más arriba; en cuanto a su clasificación considerando su tolerancia a la contaminación se utilizaron múltiples trabajos de identificación y autoecologia como Caljon (1983) y Sigee (2019), entre otros, incluyendo los utilizados para diatomeas bentónicas. La composición de los ensambles fitoplanctónicos se analizó luego de seleccionar las especies que alcanzaron más del $5 \%$ de abundancia relativa en la totalidad de las muestras y que tuvieron una frecuencia mayor al $5 \%$.

\section{Consumidores}

Para el análisis del zooplancton, se extrajeron muestras de $40 \mathrm{~L}$ de agua con una bomba sumergible integrando los sectores de agua libre y aquellos asociados con macrófitas acuáticas. Las muestras fueron filtradas por una red de $35 \mu \mathrm{m}$ de abertura de poro y fijadas con formol al 4\%. El recuento del microzooplancton $(<500 \mu \mathrm{m})$ se realizó en base a alícuotas de 1 $\mathrm{mL}$ en cámaras de Sedgewick-Rafter y para el macrozooplancton ( $>500 \mu \mathrm{m}$ ) se empleó una cámara de Bogorov de $10 \mathrm{~mL}$ (Gannon, 1971), aceptando un coeficiente de variación entre alícuotas menor al $20 \%$. Para la determinación de los taxa se utilizó bibliografía específica (Koste, 1978; Nogrady \& Segers, 2005; Reid, 1985; Ringuelet, 1958; entre otros).

Los muestreos para el análisis de la composición específica de los ostrácodos (Crustacea, Ostracoda) se realizaron de manera semicuantitativa tanto en la columna de agua como en los sedimentos de los cuerpos de agua. Para la obtención de los ejemplares en los ambientes más someros se utilizó un cucharón esmaltado en tanto que en ambientes con mayor profundidad, se usaron una red de arrastre de $35 \mu \mathrm{m}$ de abertura de poro en la columna de agua y una draga Ekman para los muestreos de sedimento. Los ejemplares fueron fijados con alcohol $70 \%$ y diseccionados para su determinación. Asimismo, el sedimento de los cuerpos de agua fue resuspendido con agua destilada y dispuesto en acuarios a fin de obtener los ejemplares eclosionados a partir de huevos de resistencia (Díaz \& Lopretto, 2017). Los individuos obtenidos por esta vía fueron utilizados para detectar presencia/ausencia de dichos estados de resistencia pero no fueron cuantificados.

\section{Descomponedores}

Para el estudio de la comunidad de hongos acuáticos se realizó la estimación de la tasa de esporulación fúngica siguiendo la metodología propuesta por Romaní et al. (2009), utilizando fragmentos de hojas de Typha latifolia (L. 1753) previamente sumergidas en los bañados durante 40 días con el objetivo de lograr una adecuada colonización fúngica (Lee \& Bukaveckas, 2002). Este procedimiento se reiteró 4 veces entre el verano e invierno de 2018. Las esporas se identificaron mediante 
claves micológicas de referencia (Seifert \& Gams, 2011). Asimismo, los hongos fueron clasificados en dos categorías fúngicas, Ingoldianos (formas estrictamente acuáticas) y dematiaceos.

\section{Análisis estadístico}

Para explorar los datos y obtener una representación gráfica de la similitud entre las muestras de los ensambles se empleó un análisis de ordenamiento multidimensional no paramétrico (nMDS; Clarke \& Warwick, 2001; Zuur et al., 2010). El nMDS se realizó a partir de los datos de abundancia (en el caso de zooplancton y hongos) y de abundancia relativa (para el fitoplancton y las diatomeas) y se utilizó una matriz de disimilitud de Bray-Curtis sobre los datos transformados con raíz cuadrada (en el caso de diatomeas, zooplancton y hongos) y $\log _{10}$ $(\mathrm{x}+1)$ (en el caso del fitoplancton). Las posibles diferencias en los diferentes ensambles fueron testeadas utilizando un análisis multivariado de similitudes de dos vías (ANOSIM), utilizando los BDF y la estacionalidad como factores, cada uno con cuatro niveles. En caso de detectarse diferencias, se realizó posteriormente una prueba SIMPER para identificar el porcentaje de contribución de cada taxón a las disimilitudes encontradas (Clarke, 1993; Clarke \& Gorley, 2006). El nMDS, el ANOSIM y el SIMPER fueron realizados utilizando el programa PRIMER 5.

\section{RESULTADOS}

\section{Productores primarios}

Se identificaron un total de 159 especies de diatomeas bentónicas (Tabla S1, material suplementario), de las cuales 130 se encontraron en el Cajaravillas, 128 en el Chubichaminí, 95 en Carnaval y 84 en Del Gato.

Al analizar el ensamble de diatomeas en el nMDS se pudo reconocer cómo las muestras se agruparon según los BDF (Figura 2; ANOSIM: $R$ Global $=0,623 ; p<0,05)$. Por un lado, se agruparon los BDF Cajaravillas y Chubichaminí y por el otro extremo se agruparon los BDF Del Gato y el Carnaval (Figura 2). El BDF Cajaravillas es el que mostró mayor agrupamiento independientemente de la estación del año, mientras que los demás bañados mostraron mayor variabilidad, pero no vinculada a la estacionalidad (Tabla 2). Se observaron diferencias significativas entre los ensambles de diatomeas entre el BDF Cajaravillas con los BDF Del Gato y Carnaval (Tabla 2).

El análisis de la clasificación de las especies de diatomeas en función de su tolerancia

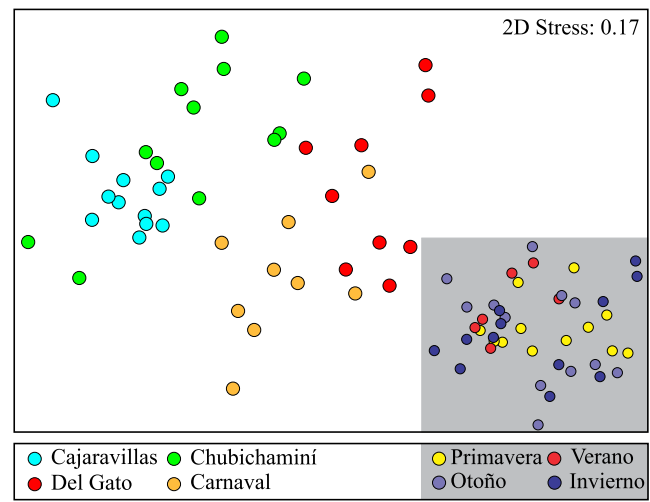

Figura 2. Representación gráfica de los dos primeros ejes del nMDS realizado en base a la abundancia relativa de los ensambles de diatomeas bentónicas entre los cuatro BDF. En gris, el mismo ordenamiento nMDS realizado respecto a las estaciones del año.

Figure 2. Graphic representation of the first two axes of the nMDS based on the relative abundance of the benthic diatoms assemblage's among the four BDFs. In gray, the same nMDS made with respect to the seasons of the year.

a la contaminación reveló que los mayores porcentajes de especies sensibles se registraron en el Cajaravillas (11\%) y Chubichaminí (9\%), siendo las principales representantes Achnanthidium exiguum (Grunow) Czarn 1994, Bacillaria paxillifera (O.F. Mull.) N.I. Hendey 1951 y Navicula kotschy Grunow 1860. Si bien las especies muy tolerantes (Nitzschia palea (Kützing) W.Sm. 1856, Gomphonema parvulum (Kützing) Kützing 1849, Nitszchia amphibia Grunow 1862 y Lemnicola hungarica (Grunow) Round \& Basson 1997) presentaron porcentajes elevados en todos los bañados, en el BDF Del Gato el valor superó el $80 \%$.

En el fitoplancton se identificaron 174 taxa en los BDF estudiados (Tabla S2, material suplementario), de los cuales se encontraron 90 en el Chubichaminí, 88 en el Cajaravillas, 64 en Del Gato y solo 30 en el Carnaval. Muchos 
Tabla 2. Comparaciones a posteriori del ANOSIM ( $\left.{ }^{*} p<0,05\right)$ entre BDF, porcentaje de disimilitud promedio y especies que más aportan a esas diferencias como resultado del SIMPER para el ensamble de diatomeas bentónicas, fitoplancton, zooplancton y hongos acuáticos.

Table 2. A posteriori comparison of the ANOSIM ( ${ }^{*} p<0.05$ ) between BDFs, average dissimilarity percentage and species that most contribute to these differences as a result of the SIMPER for the benthic diatoms, phytoplankton, zooplankton and aquatic fungi assemblage.

\begin{tabular}{|c|c|c|c|c|c|c|}
\hline & $\begin{array}{l}\text { Baña } \\
\text { compa }\end{array}$ & $\begin{array}{l}\text { dos } \\
\text { rados }\end{array}$ & & ANOSIM & & SIMPER \\
\hline & 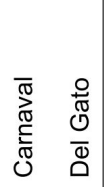 & 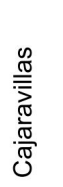 & $\begin{array}{l}. \overline{\frac{E}{E}} \\
\frac{1}{0} \\
\frac{c}{0} \\
\frac{.0}{0} \\
\frac{D}{D} \\
\frac{1}{U}\end{array}$ & $\mathrm{R}$ & $\begin{array}{l}\frac{a}{0} \\
\frac{0}{2} \\
\frac{\underline{2}}{\bar{E}} \\
\frac{0}{0} \\
\frac{0}{0}\end{array}$ & Contribución (especies destacadas) \\
\hline 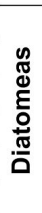 & 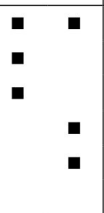 & $\begin{array}{l}. \\
\text {. } \\
\text {. }\end{array}$ & . & $\begin{array}{l}0,39 \\
\mathbf{0 , 8 6 ^ { \star }} \\
0,62 \\
\mathbf{0 , 9 2} \\
0,59 \\
0,40\end{array}$ & $\begin{array}{l}56 \\
59 \\
60 \\
67 \\
63 \\
53\end{array}$ & $\begin{array}{l}\text { Nitzschia palea (6\%) } \\
\text { Lemnicola hungarica }(\mathbf{4} \%) \text {, Melosira varians (3\%) } \\
\text { N. palea }(5 \%), \text { M. varians }(3,5 \%) \\
\text { N. palea }(5 \%) \\
\text { N. palea }(5 \%), \text { Pseudofallacia monoculata }(4 \%) \\
\text { N. palea }(5 \%), \text { L. hungarica }(4 \%)\end{array}$ \\
\hline $\begin{array}{l}\frac{5}{0} \\
\frac{0}{0} \\
\frac{\pi}{\pi} \\
\frac{0}{0} \\
\frac{2}{2} \\
\frac{1}{1}\end{array}$ & $\begin{array}{ll} & \text { I } \\
\text {. } & \\
\text {. } & \\
& \text {. }\end{array}$ & $\begin{array}{l}. \\
\text { - }\end{array}$ & - & 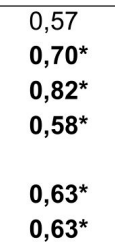 & $\begin{array}{l}91 \\
91 \\
86 \\
99\end{array}$ & $\begin{array}{l}\text { Jaaginema subtilissimum (12\%), Euglena oxyuris }(9 \%) \\
\text { J. subtilissimum }(9 \%) \text {, Komvophoron minutum }(6 \%) \\
\text { J. subtilissimum }(12,5 \%), \text { K. minutum }(9 \%) \\
\text { Trachelomonas planctonica }(8 \%), \text { Komvophoron constrictum } \\
\text { (6) } \\
\text { E. oxyuris }(11 \%), \text { Phacus longicauda }(9 \%) \\
\text { P. longicauda }(7 \%), \text { T. planctónica }(6 \%)\end{array}$ \\
\hline $\begin{array}{l}5 \\
0 \\
0 \\
\frac{0}{\pi} \\
\frac{\pi}{0} \\
\frac{0}{0} \\
\text { N }\end{array}$ & $\begin{array}{ll}. & \\
. & \\
. & \end{array}$ & $\begin{array}{l}. \\
\text {. }\end{array}$ & - & 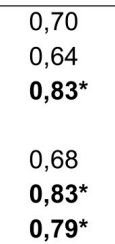 & $\begin{array}{l}81 \\
73 \\
89 \\
79 \\
88 \\
83\end{array}$ & $\begin{array}{l}\text { Prorodon sp. }(13 \%) \\
\text { Muchas especies (4\% aprox c/u). } \\
\text { Larvas nauplii (14\%), Centropyxis aculeata }(4 \%) \text {, Keratella } \\
\text { tropica }(4 \%) \\
\text { Prorodon sp. }(13 \%) \\
\text { Prorodon sp. y larvas nauplii ( } 20 \% \text { en conjunto) } \\
\text { Larvas nauplii, } K \text {. tropica y Filinia longiseta ( } 16 \% \text { en conjunto) }\end{array}$ \\
\hline $\begin{array}{l}\text { 오 } \\
\text { 옴 } \\
\text { 움 }\end{array}$ & $\begin{array}{ll} & \text { a } \\
\text {. } & \\
\text { घ } & \\
& \text {. }\end{array}$ & $\begin{array}{l}\text {. } \\
\text { - }\end{array}$ & - & 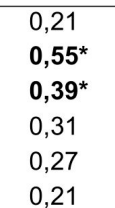 & $\begin{array}{l}60 \\
66 \\
68 \\
60 \\
63 \\
61\end{array}$ & $\begin{array}{l}\text { Margaritispora aquatica (8\%), Amniculicola longissima (8\%) } \\
\text { Zalerion sp. }(\mathbf{9} \%) \text {, A. Iongissima (9\%) } \\
\text { A. longissima (11\%), M. aquatica (10) } \\
\text { M. aquatica }(8 \%), \text { Zalerion sp. }(7 \%) \\
\text { M. aquatica }(10 \%), \text { A. Iongissima }(9 \%) \\
\text { A. longissima }(10 \%), \text { M. aquatica }(8 \%)\end{array}$ \\
\hline
\end{tabular}

de estos taxa tuvieron muy baja frecuencia y abundancia relativa, por lo que solo resultaron seleccionados 84 para los análisis. Al analizar los grupos fitoplanctónicos dominantes, se observó que cada BDF tuvo un grupo taxonómico distintivo (Figura 3); en el Cajaravillas dominaron las clorofitas clorococales representadas principalmente por Crucigenia quadrata Morren, Dictyosphaerium pulchellum Wood y Didimocystis bicellularis (Chodat) Komárek. En el BDF Chubichaminí dominaron las euglenofitas, tanto loricadas (pertenecientes a los géneros Strombomonas Deflandre y Trachelomonas Ehrenberg) como no loricadas (pertenecientes a los géneros Euglena Ehrenberg, Lepocinclis Perty y Phacus Dujardin).

En el BDF Del Gato dominaron las diatomeas pennales como Nitzschia palea, y en el BDF Carnaval las cianobacterias filamentosas como Jaaginema subtilissimum (Kützing ex De Toni) Anagnostidis et Komárek y Phormidium articulatum (Gardner) Anagnostidis et Komárek. Como resultado del nMDS realizado en base a la composición específica del fitoplancton, los BDF Chubichaminí y Carnaval fueron los más disimiles y se ubicaron en los dos extremos del grafico (Figura 4). Entre estos extremos se encuentran el Cajaravillas, más cercano al Chubichaminí y Del Gato, más cercano al Carnaval (Figura 4). De acuerdo con el resultado 
del ANOSIM los ensambles fitoplanctónicos de los BDF se separaron significativamente ( $R$ Global $=0,616, p<0,05)$ y, en las comparaciones a posteriori, la mayor diferencia correspondió a los BDF Chubichaminí y Carnaval. En cuanto a la estacionalidad hubo diferencias menos marcadas ( $R$ Global=0,426; $p<0,05$ ) y en las comparaciones a posteriori solo fueron significativas las muestras de otoño y primavera $(R=0,731 ; p<0,05 ;$ Tabla 2).

Al analizar la disimilitud (SIMPER) entre BDF, los porcentajes siempre fueron muy elevados, superiores al 75\% (Tabla 2), y estas diferencias en general fueron aportadas por muchas especies (entre 19 y 29) con una baja contribución individual (máximo 12,5\%).

Al analizar las especies en relación a su modo de vida, se observó que la proporción de algas de hábitos euplanctónicos con respecto a las ticoplanctónicas fueron superiores en los BDF Cajaravillas (82,7\%) y Chubichaminí $(66,8 \%)$ que en los BDF Del Gato y Carnaval, donde dominaron las algas ticoplanctónicas, llegando casi al $100 \%$ en el Carnaval.

En cuanto a la tolerancia de las especies a la contaminación orgánica y la eutrofización se observó el predominio de las especies

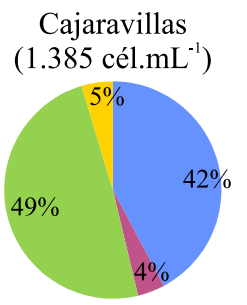

Del Gato (192 cél. $\left.\mathrm{mL}^{-1}\right)$

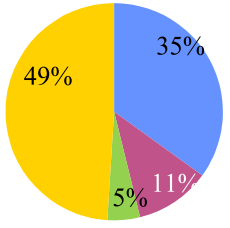

-Cyanophyta Euglenophyta $\backsim$ Dinoflagellata Chlorophyta Bacillariophyta

Figura 3. Densidad fitoplanctónica: Relación porcentual promedio de los grandes grupos taxonómicos para cada BDF. Entre paréntesis: densidad total en promedio anual.

Figure 3. Phytoplankton density: Average percentage ratio of the main taxonomic groups for each BDF. In brackets: total annual average density.

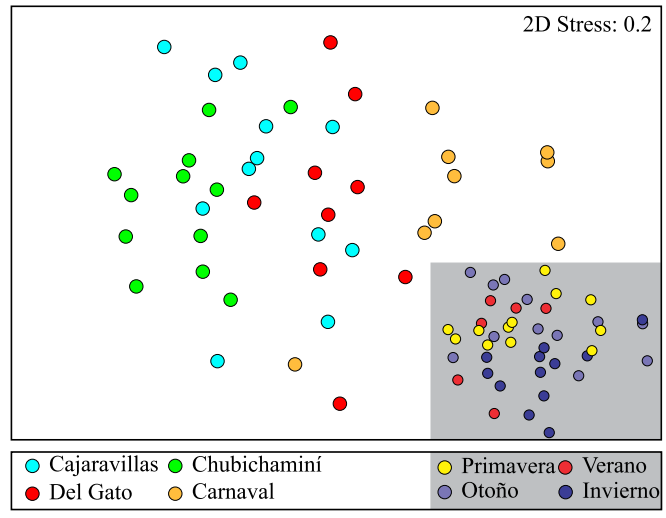

Figura 4. Representación gráfica de los dos primeros ejes del nMDS realizado en base a la abundancia relativa de los ensambles de fitoplancton entre los cuatro BDF. En gris, el mismo ordenamiento del nMDS realizado respecto a las estaciones del año.

Figure 4. PGraphic representation of the first two axes of the nMDS based on the relative abundance of the phytoplankton assemblage's among the four BDFs. In gray, the same nMDS made with respect to the seasons of the year.

tolerantes en el BDF Cajaravillas $(77,1 \%)$ y el Chubichaminí (48,7\%). Por otro lado, las especies muy tolerantes dominaron en los BDF Del Gato (83,3\%) y Carnaval (97,4\%). Con relación a las especies sensibles el mayor porcentaje se observó en el BDF Chubichaminí (8\%).

\section{Consumidores}

En el zooplancton de los cuatro BDF se registró un total de 145 especies (Tabla S3 material suplementario), con representantes de rizópodos, amebozoos, ciliados, rotíferos, cladóceros, copépodos y grupos menos diversos y abundantes como gastrotricos, anélidos y tardígrados, entre otros.

Cada BDF tuvo un ensamble distintivo. En el BDF Del Gato dominaron los ciliados, seguidos por los amebozoos; mostrando que más del 80 $\%$ de las especies fueron protistas (Figura 5). La estructura del zooplancton en los BDF Carnaval y Cajaravillas fue semejante entre sí, con un predominio de rotíferos y amebas testáceas. Sin embargo, particularmente en el Cajaravillas, aparecieron en tercer lugar los copépodos y los protistas fueron menos abundantes; mientras que en el Carnaval el porcentaje de protistas siguió siendo elevado y los copépodos 
solo alcanzaron el 2\%. En el Chubichaminí la composición del ensamble zooplanctónico fue radicalmente diferente, con una codominancia de copépodos y rotíferos.

El nMDS del zooplancton mostró que los ensambles de los diferentes BDF se separaron según los sitios de estudio, pero no siguiendo las estaciones climáticas (Figura 6). Los BDF Chubichaminí y el Carnaval se ubicaron en los extremos, en tanto que los BDF Cajaravillas y Del Gato conformaron grupos intermedios o de transición entre los dos antes mencionados. Las muestras correspondientes a los BDF Carnaval y Cajaravillas fueron las más cercanas entre sí, mostrando que los ensambles fueron menos heterogéneos y con mayor porcentaje de similitud (Tabla 2).

EI ANOSIM mostró los ensambles fueron diferente entre los BDF ( $R$ Global $=0,713 ; p<$ 0,05 ) y las estaciones del año ( $R$ Global $=0,65 ; p<$ $0,05)$. Las comparaciones a posteriori revelaron que el ensamble del Chubichaminí fue diferente a todos los demás (Tabla 2). Este, juntamente con el Del Gato, fueron los más heterogéneos (\% de similitud muy bajos) y entre ellos la diferencia fue máxima (88\%) y estuvo promovida mayormente por el predominio del ciliado del género Prorodon sp. Ehrenberg y larvas nauplii

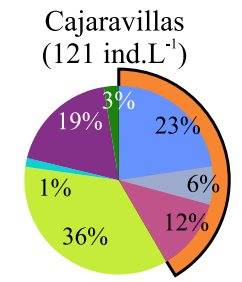

Del Gato

(444 ind. . $^{-1}$ )

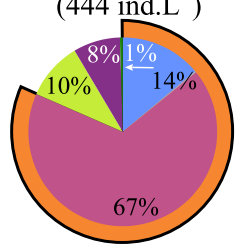

Amoebozoa $\backsim$ Rizharia $₫$ Ciliophor

Rotifera $\backsim$ Branchiopoda $\backsim$ Maxillopoda (5) Protista $=$ Otros grupos

Figura 5. Densidad zooplanctónica: Relación porcentual promedio de los grandes grupos taxonómicos para cada BDF. Entre paréntesis: densidad total en promedio anual. El arco anaranajado señala a los protistas.

Figure 5. Zooplankton density: Average percentage ratio of the main taxonomic groups for each BDF. In brackets: total annual average density. Orange arc points to protists.

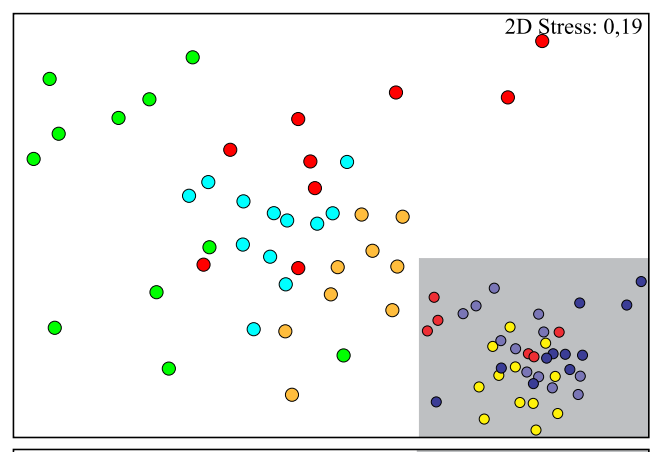

○ Cajaravillas ○ Chubichaminí $\bigcirc$ Primavera $\bigcirc$ Verano - Del Gato $\bigcirc$ Carnaval Otoño Invierno

Figura 6. Representación gráfica de los dos primeros ejes del nMDS realizado en base a la abundancia relativa de los ensambles de zooplancton entre los cuatro BDF. En gris, el mismo ordenamiento del nMDS realizado respecto a las estaciones del año.

Figure 6. Graphic representation of the first two axes of the nMDS based on the relative abundance of the zooplankton assemblage's among the four BDFs. In gray, the same nMDS made with respect to the seasons of the year.

de copépodos.

Entre los ostrácodos, la distribución de las especies en los BDF fue muy heterogénea. Se identificaron nueve especies pertenecientes a cinco familias (Figura 7 y Tabla S4, material suplementario). La familia Cyprididae fue la más representada con cinco especies, mientras que las restantes familias contaron con una sola especie cada una. Chlamydotheca incisa (Claus, 1892) es la única especie común a los cuatro BDF, mientras que las especies de los géneros Candona (Baird, 1846), Herpetocypris (Claus, 1892) y Darwinula (Brady \& Norman, 1889) estuvieron presentes solo en los BDF Cajaravillas y Chubichaminí; en tanto que Ilyocypris ramirezi (Cusminsky \& Whatley, 1996) fue hallada exclusivamente en el BDF Del Gato.

\section{Descomponedores}

Se registraron un total de 76 taxa de hongos acuáticos ingoldianos y dematiaceos (Tabla S5, material suplemetario), siendo Ascomycota el phylum predominante. Con respecto a las abundancias de hongos ingoldianos (hialinos) los BDF Chubichaminí (63\%) y Carnaval (78\%) tuvieron mayores valores a diferencia del BDF Cajaravillas (34\%). En este último los hongos dematiaceos (pigmentados) alcanzaron la 


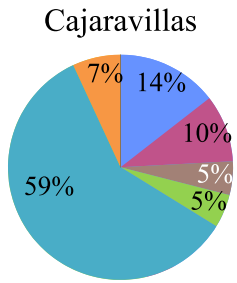

Del Gato

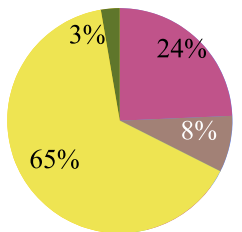

-Candona sp.๘Chlamydotheca incisa - Cypridoidea indet. $=$ Cypridopsis vidua -Cytheridella ilosvayi Darwinula sp. Heretocypris incongruens - Herpetocypris sp. Ilyocypris ramirezi

Figura 7. Importancia relativa de las especies de ostrácodos encontrados en los cuatro BDF.

Figure 7. Relative importance of the ostracod species found in the four BDFs.

mayor abundancia (66\%). Por otro lado, en el BDF Del Gato las abundancias fueron similares para ambos tipos fúngicos (50\%).

El análisis de nMDS evidenció que los ensambles de hongos de los BDF Cajaravillas y Chubichaminí se diferenciaron levemente del Carnaval y Del Gato. Por otro lado, se observó una separación temporal entre las muestras del verano con las de otoño e invierno (Figura 8).

El análisis de ANOSIM (Tabla 2) mostró diferencias en la composición de los ensambles ( $\mathrm{R}$ Global = 0,305; $p=0,01$ ). A partir del análisis SIMPER, se observó que los BDF tuvieron una similitud promedio de entre un $40 \%$ y $45 \%$. Los taxa que más contribuyeron a la similitud de muestras en cada BDF, fueron Arthrinium sp. Kunze, Zalerion sp. R.T.Moore \& Meyers, Diplococcium sp. Grove y Torula sp. Pers. No obstante, el bañado Chubichaminí se diferenció por la contribución de otras especies, Margaritispora aquatica Ingold (1942) y Amniculicola longissima (Sacc. \& P. Syd.) Nadeeshan \& K.D. Hyde (2016). Las mayores diferencias (disimilitudes) se encontraron entre los BDF Carnaval respecto al Chibichaminí y Cajaravillas (Tabla 2).

\section{DISCUSIÓN}

Los humedales ubicados en la llanura de

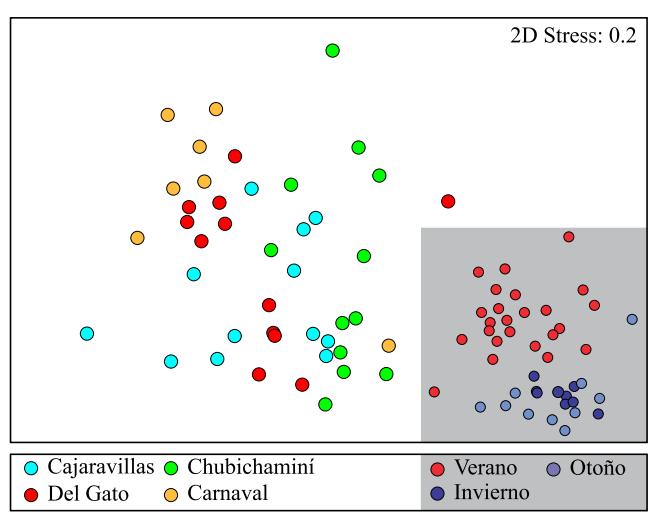

Figura 8. Representación gráfica de los dos primeros ejes del nMDS realizado en base a la abundancia relativa de las esporas de hongos acuáticos entre los cuatro BDF. En gris, el mismo ordenamiento del nMDS realizado respecto a las estaciones del año.

Figure 8. Graphic representation of the first two axes of the nMDS based on the relative abundance of the aquatic fungal spores among the four BDFs. In gray, the same nMDS made with respect to the seasons of the year.

inundación de los cursos de agua mantienen una gran diversidad de organismos debido a la heterogeneidad del hábitat, las fluctuaciones hidrológicas y la calidad de sus aguas (Rodrigues et al., 2015). Los cambios en el uso del suelo, como el aumento del área dedicada a la agricultura fertilizada, suponen una amenaza para estos ambientes que afecta a los organismos que los habitan (Bussi et al., 2016; 2017). De este modo se generan modificaciones en los ecosistemas acuáticos que son evidenciadas a través de las comunidades (Allan, 2004). Según los resultados obtenidos, las respuestas de la microbiota mostraron variaciones con relación a la calidad del agua y a la variabilidad hidrológica que condicionó la permanencia del agua en los BDF. Se identificaron patrones de respuesta similares en los ensambles de todos los grupos estudiados (diatomeas bentónicas, fitoplancton, zooplancton y hongos) que permitieron diferenciar los BDF ubicados en zonas rurales de aquéllos ubicados en áreas periurbanas; en tanto que la variación temporal dada por la estacionalidad climática no fue determinante en los ordenamientos para la mayoría de los grupos. En los BDF ubicados en zonas rurales la comunidad planctónica estuvo representada por una mayor diversidad de grandes grupos taxonómicos. Así, el fitoplancton estuvo integrado en forma relativamente 
equitativa por cianobacterias, euglenofitas, clorofitas y diatomeas, con la presencia de dinoflagelados en el BDF Chubichamini y el zooplancton por protozoos, rotíferos, copépodos y cladóceros. En contraste, en los BDF ubicados en áreas periurbanas, el plancton estuvo representado por pocos grupos, el fitoplancton por cianobacterias o diatomeas, $y$ el zooplancton por protistas (ciliados o tecamebas). En estos últimos ambientes se halló además un mayor porcentaje de especies que se encuentran en el plancton de manera ocasional (ticoplanctónicas) y que, en su mayoría, proceden de la comunidad bentónica y epifítica, como es el caso de las cianobacterias filamentosas y de las diatomeas bentónicas. Uno de los factores que limita el desarrollo de los microorganismos es el régimen hidrológico, que determina la permanencia del agua superficial. Esto tiene estrecha vinculación con el nivel freático, cuyo descenso debido a la sobreexplotación de los acuíferos afecta a los BDF ubicados en zonas periurbanas (Rodrigues Capítulo et al., 2020). Así, los grupos con ciclos de vida más largos y complejos como los microcrustáceos encuentran condiciones ambientales más adversas en este tipo de ambientes, en contraposición a los protistas que dominaron en estos BDF favorecidos por tiempos generacionales más cortos, de uno o dos días y una elevada capacidad reproductiva, lo que permiten una extremada rapidez en la sustitución de especies (Andrushchyshyn et al., 2003; Finlay \& Esteban, 1998) y constituyen una estrategia exitosa ante condiciones hidrológicas inestables. Por otra parte, en los mismos BDF el predominio de microorganismos de origen bentónico en el plancton constituye una respuesta a la escasa profundidad. En este sentido, es conocido que las tecamebas adquieren gran importancia por el aporte de especies litorales y bentónicas de los géneros Difflugia Leclerc, 1815, Centropyxis Stein, 1857 y Euglypha Dujardin, 1840 que acceden al plancton (Modenutti, 1991).

Las diferencias en el uso del suelo y consecuentemente en el aporte de nutrientes y agroquímicos a los bañados también se vieron reflejadas en la simplificación de los niveles tróficos que componen los ensambles de microorganismos (Figura 9). En tal sentido, el zooplancton de los BDF ubicados en la zona periurbana estuvo dominado por protistas principalmente consumidores de bacterias, lo que expresa un predominio de la vía detritívora. Por el contrario, en los BDF ubicados en el zonas rurales la representación de los diversos niveles tróficos fue más equitativa, con presencia de bacteriófagos (la mayoría de los protistas presentes), consumidores de algas nanoplanctónicas (filtradores y capturadores) y depredadores, que estuvieron ausentes en los BDF de ubicación periurbana. Una simplificación de la estructura trófica como la observada en los BDF periurbanos implica limitaciones en el reciclado de la materia y consecuentemente influye negativamente en los procesos de autodepuración del agua (Haberman \& Haldna, 2014; Jeppesen et al., 2011).

Las inundaciones periódicas favorecen el desarrollo de organismos que son transportados por los ríos y desplazados hacia el valle aluvial (Junk et al., 1989), donde también quedan depositadas sus estructuras de resistencia (Solari et al., 2018; Zaplara et al., 2018). Los pulsos de inundación, que pueden durar días o semanas, permiten que los estados de resistencia eclosionen contribuyendo así a la capacidad de recuperación de los cuerpos de agua de la llanura de inundación (Brock et al., 2003), por lo que constituyen una reserva de material genético cuya viabilidad es de gran importancia ecológica para el ecosistema (McQuoid et al., 2002). Muchas de las especies halladas en este estudio presentan estrategias de este tipo. Por ejemplo, las diatomeas generan esporas de reposo que pueden activarse luego de pasar por un periodo de sequía (Round, 1990) e incluso algunas pueden permanecer viables durante varias décadas (Stockner \& Lund, 1970), tal el caso de representantes de los géneros Achnanthes Bory, 1822; Luticula D.G. Mann, 1990; Pinnularia C.G. Ehrenberg, 1843; Mayamaea H. Lange-Bertalot, 1997 y Hantzschia A. Grunow, 1877 (Souffreau et al., 2013), hallados en los BDF. En el fitoplancton también se reportaron taxa capaces de sobrevivir bajo condiciones de déficit hídrico por la producción de estados de reposo (acinetos) tales como las especies del género Anabaena Bory ex Bornet \& Flahault o por estados de latencia, como las de los géneros Pediastrum Meyen, Eudorina Ehrenberg y Ulothrix 
Kützing (Coleman, 1983; Ellegaard et al., 2013, Ellegaard \& Ribeiro, 2018). Otro de los taxa adaptados a esta condición que se observó fue Euglena viridis (O.F. Müller) Ehrenberg, que produce quistes (Hindák et al., 2000).

Entre los consumidores, muchos de los ciliados (Vorticella Linnaeus, 1767, Euplotes Ehrenberg, 1830; Halteria Dujardin, 1841, Epistylis Ehrenberg, 1830) junto con rotíferos bdelloideos hallados en este estudio pueden aparecer tempranamente en el agua de inundación ya que habitan normalmente en el agua intersticial del suelo (Foissner et al., 1999; Schwarz \& Frenzel, 2003). Además, los rotíferos digononta también presentan anhidrobiosis (Battauz, 2015). La producción de huevos resistentes a la sequía es generalizada en rotíferos monogononta y cladóceros, y está presente en copépodos calanoideos (Vijverberg, 1977). Por su parte, Acanthocyclops robustus (Sars) (Copepoda, Cyclopoida) presenta diapausa sin enquistamiento (Dahms, 1995). Entre los ostrácodos, las hembras de Chlamydotheca incisa, Heterocypris incongruens (Ramdohr, 1808) y Cypridopsis vidua (O.F. Müller, 1776) tienen la capacidad de producir huevos de resistencia, mejorando la supervivencia y la dispersión (Spencer \& Blaustein, 2001). Además, la presencia de una cámara de incubación en la parte posterior del caparazón en especies como Cytheridella ilosvayi Daday, 1905 permite retener huevos y los primeros estadios juveniles.

En relación con los hongos acuáticos, algunas especies identificadas en los BDF, tales como Alatospora acuminata Ingold (1942), Amniculicola Iongissima, Tetracladium marchalianum De Wild (1893) y Lemonniera sp. De Wild., son capaces de sobrevivir bajo las condiciones adversas que genera la sequía (Sanders \& Webster, 1978; Sati \& Belwal, 2005).

El mayor deterioro de la calidad del agua observado en los BDF de ubicación periurbana (Del Gato y Carnaval) se correspondió con porcentajes más elevados de especies fitoplanctónicas consideradas muy tolerantes a la contaminación orgánica y a la eutrofización lo que coincide con lo reportado por Bauer (2009) para arroyos periurbanos del noreste de la llanura pampeana. Un patrón similar fue observado en los ensambles de diatomeas bentónicas advirtiéndose un mayor porcentaje de taxa muy tolerantes y una reducción de los sensibles en los BDF ubicados en la zona periurbana. Por otra parte, el predominio de zooplancton de pequeña talla en los BDF ubicados en zonas periurbanas se debería a una mayor concentración de nutrientes, según lo reportado por (Jeppesen et al., 2011). Entre los ostrácodos, la presencia de los géneros más sensibles a la contaminación, Candona, Darwinula y Herpetocypris, se verificó en los bañados ubicados en el área rural. También los descomponedores mostraron la misma tendencia con la presencia de especies sensibles a la contaminación, entre las que se halla Amniculicola longissima (Solé et al., 2008; Tarda et al., 2019).

El avance de la presión humana sobre estos ambientes debido a las prácticas agropecuarias y la urbanización, sumado a la sobreexplotación de los acuíferos, conduce a la retracción de los mismos y con ello a la desaparición de superficies en los valles aluviales capaces de albergar inóculos que permitan el rejuvenecimiento de la biota durante los pulsos hidrológicos. Estas pérdidas han sido documentadas en otras latitudes, e incluso desde hace tiempo se realizan esfuerzos en el desarrollo y la implementación de la restauración en ambientes de este tipo como señalan Dodson \& Lillie (2001) y Mitsch \& Wilson (1996) en Estados Unidos de América. De este modo, se debe remarcar que es fundamental tener en cuenta los importantes servicios ecosistémicos que estos ambientes brindan en la planificación del uso del territorio. El albergue de organismos tanto vivos como en forma de propágulos, la depuración del agua por medio de la mineralización de materia orgánica y de la retención de contaminantes inorgánicos, la amortiguación de los pulsos de inundación, e incluso las posibilidades de uso recreativo son sólo algunos de los beneficios que se pierden cuando estos ambientes son degradados o destruidos.

\section{CONCLUSIONES}

Los resultados obtenidos muestran que, a partir del estudio de los ensambles de microorganismos y sus respuestas ante las variaciones ambientales (Figura 9), es 

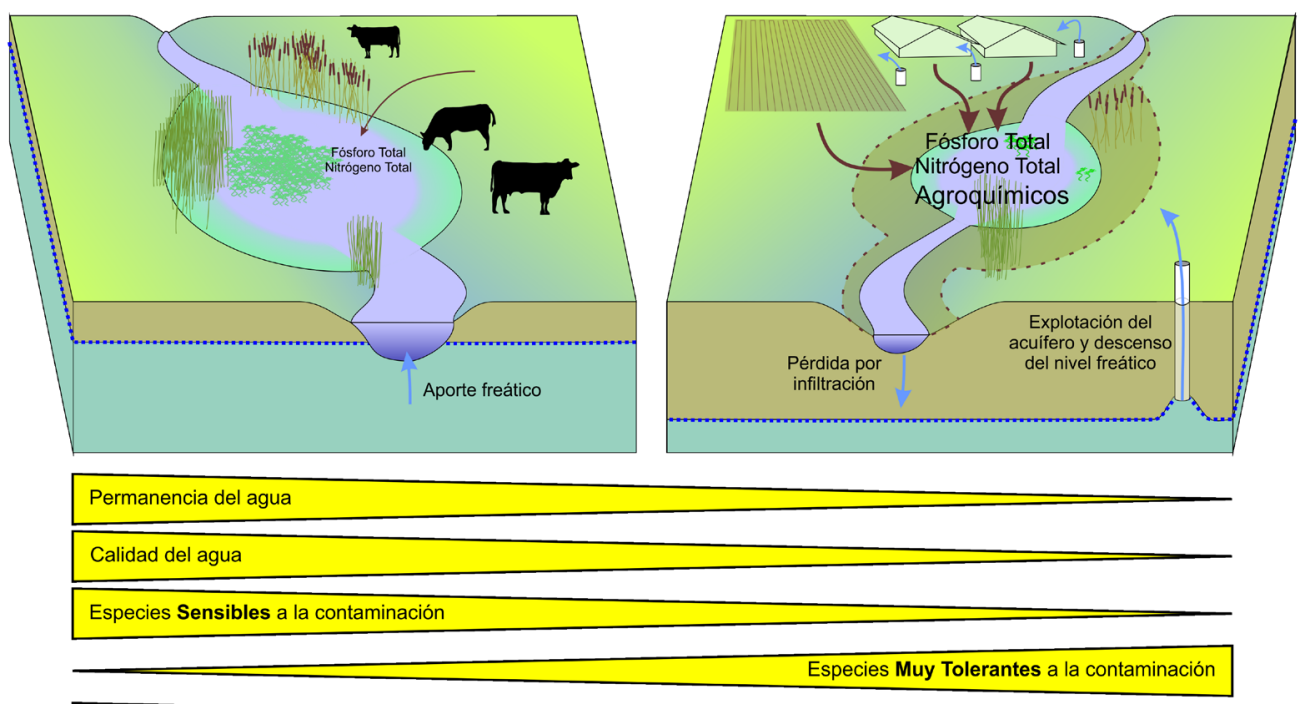

Complejidad de las tramas tróficas

Figura 9. Esquema conceptual sobre algunas de las características ambientales de ambos tipos de BDF estudiados y los principales resultados biológicos obtenidos. A la izquierda un BDF de ubicación rural y a la derecha uno de ubicación periurbana.

Figure 9. Conceptual framework of some of the environmental characteristics of both types of BDF studied and the main biological results obtained. On the left a rural BDF and on the right a peri-urban location BDF.

posible diferenciar los BDF ubicados en áreas rurales con uso del suelo principalmente dedicado a ganadería extensiva, con mayor superficie, volumen y profundidad (Cajaravillas y Chubichaminí), con mejor calidad del agua y del hábitat, caracterizados por tramas tróficas más complejas y con mejor representatividad de distintos niveles tróficos (predominio de la vía fotosintética) y con más especies sensibles a la contaminación; de los ubicados en áreas periurbanas con actividad agrícola $u$ hortícola, con menores superficies, volumen y profundidad y desvinculados del agua freática (Del Gato y Carnaval), con tramas tróficas más simplificadas con preponderancia del bucle microbiano y con más cantidad de especies muy tolerantes a la contaminación. De este modo, el estudio de los microorganismos de los bañados de desborde fluvial constituye una herramienta valiosa para alertar sobre los cambios ambientales y los diferentes impactos a los que están expuestos.

\section{AGRADECIMIENTOS}

A Noelia Ferrando y Roberto Jensen por su ayuda en el campo para la toma de muestras biológicas; a Cristina Claps por su valioso aporte en la identificación de organismos del zooplancton.

Este trabajo fue posible gracias al subsidio de la Agencia Nacional de Promoción Científica y Tecnológica (ANPCyT), PIP 2015-1342 N. Gómez. Nº1177 de Contribución del ILPLA.

\section{REFERENCIAS}

Allan, J. D. (2004). Landscapes and riverscapes: the influence of land use on stream ecosystems. Annual Review of Ecology, Evolution, and Systematics, 35, 257-284 https://doi.org/10.1146/ annurev.ecolsys.35.120202.110122. 
Andrushchyshyn, O., Magnusson, A. K. \& Williams, D. D. (2003). Ciliate populations in temporary freshwater ponds: seasonal dynamics and influential factors. Freshwater Biology, 48(3), 548-564. https://doi.org/10.1046/j.13652427.2003.01028.x

Battauz, Y. S. (2015). Propágulos del zooplancton: importancia en el mantenimiento de la diversidad en los ambientes acuáticos y dispersión de las especies. (Tesis Doctoral, Facultad de Bioquímica y Ciencias Biológicas, UNL).

Bauer, D. E. (2009). Ecología del fitoplancton de arroyos pampeanos y su valor como indicador de la calidad del agua. (Tesis Doctoral, Facultad de Ciencias Naturales y Museo, UNLP).

Bayley, P. B. (1995). Understanding large river: floodplain ecosystems. Bioscience, 45, 153-158.

Brinson, M. M., Hauer, F. R., Lee, L. C., Nutter, W. L., Rheinhardt, R. D., Smith, R. D. \& Whigham, D. F. (1995). A guidebook for application of hydrogeomorphic assessments to riverine wetlands. Technical Report WRP-DE-11, Waterways Experiment Station. Mississippi, USA: Army Corps of Engineers.

Brock, M. A., Nielsen, D. L., Russell, J. S., Green, J. D. \& Langley, J. D. (2003). Drought and aquatic community resilience: the role of eggs and seeds in sediments of temporary wetlands. Freshwater Biology, 48, 1207-1218.

Bruce, L. C., Hamilton, D., Imberger, J., Gal, G., Gophen, M., Zohary, T. \& Hambright, K.D. (2006). A numerical simulation of the role of zooplankton in C, N and P cycling in Lake Kinneret, Israel. Ecological Modelling, 93, 412-436. https:// doi.org/10.1016/j.ecolmodel.2005.09.008

Bussi, G., Whitehead, P. G., Bowes, M. J., Read, D. S., Prudhomme, C. \& Dadson, S. J. (2016). Impacts of climate change, land-use change and phosphorus reduction on phytoplankton in the River Thames (UK). Science of the Total Environment, 572, 1507-1519. https://doi. org/10.1016/j.scitotenv.2016.02.109

Bussi, G., Janes, V., Whitehead, P. G., Dadson, S. J. \& Holman, I. P. (2017). Dynamic response of land use and river nutrient concentration to long-term climatic changes. Science of the Total Environment, 590, 818-831. https://doi. org/10.1016/j.scitotenv.2017.03.069
Caljon, A. G. (1983). Developments in Hydrobiology Brackish-water Phytoplankton of the Flemish Lowland. The Hague: Dr W. Junk Publisher.

Clarke, K. R. (1993). Nonparametric multivariate analyses of changes in community structure. Australian Journal of Ecology, 18(1), 117-143. https://doi.org/10.1111/j.1442-9993.1993. tb00438.x

Clarke, K. R. \& Gorley, R. N. (2006). Primer. PRIMER-e, Plymouth.

Clarke, K. R., \& Warwick, R. M. (2001). A further biodiversity index applicable to species lists: variation in taxonomic distinctness. Marine Ecology Progress Series, 216, 265-278. https:// doi.org/doi:10.3354/meps216265.

Cochero, J., Di Giorgi, H., Donadelli, J. L., Suárez, J., Simonetti, R., Finkler, N. R y Gasparini Fernandes Cunha, D. (2020). El rol de los bañados de desborde fluvial en la retención de nutrientes y su actividad metabólica. Biología Acuática, 35.

Coleman, A. W. (1983). The roles of resting spores and akinetes in chlorophyte survival. In: Fryxell, G. A. (Ed.). Survival strategies of the algae ( $p p$ 1-21). London: Cambridge University Press.

Dahms, H. U. (1995). Dormancy in the Copepodaan overview. Hydrobiologia, 306(3), 199-211. https://doi.org/10.1007/BF00017691

Davidson, N. C. (2014). How much wetland has the world lost? Long-term and recent trends in global wetland area. Marine and Freshwater Research, 65(10), 936-941. https:// doi.org/10.1071/MF14173

de Jonge, V. (1979). Quantitative separation of benthic diatoms from sediments using density gradient centrifugation in the colloidal silica Ludox-TM. Marine Biology, 51, 267-278. https:// doi.org/10.1007/BF00386807

Desikachary, T. V. (1950). Cyanophyta. New Delhi: Indian Council of Agricultural Research.

Descy, J-P. \& Coste, M. (1991). A test of methods for assessing water quality based on diatoms. Internationale Vereinigung für Theoretische und Angewandte Limnologie: Verhandlungen, 24, 2112-2116. https://doi.org/10.1080/03680770 .1989 .11899905 
Díaz, A. R, \& Lopretto, E. C. (2017). Postembryonic development of nonmarine ostracod Chlamydotheca arcuata (Sars, 1901) (Crustacea: Ostracoda), reared in the laboratory. Turkish Journal of Zoology, 41(2), 209-226.

Dodson, S. I., Arnott, S. E. \& Cottingham, C. L. (2000). The relationship in lake communities between primary productivity and species richness. Ecology, 81, 2662-2679. https://doi. org/10.1890/0012-9658(2000)081[2662:TRILC B]2.0.CO;2

Dodson, S. I., \& Lillie, R. A. (2001). Zooplankton communities of restored depressional wetlands in Wisconsin, USA. Wetlands, 21(2), 292-300. https://doi.org/10.1672/02775212(2001)021[0292:ZCORDW]2.0.CO;2

Ellegaard, M. \& Ribeiro, S. (2018). The long $\square$ term persistence of phytoplankton resting stages in aquatic 'seed banks'. Biological Reviews, 93(1), 166-183. https://doi.org/10.1111/brv.12338

Ellegaard, M., Ribeiro, S., Lundholm, N., Andersen, T. J., Berge, T., Ekelund, F. \& Godhe, A. (2013). Using the sediment archive of living dinoflagellate cysts and other protist resting stages to study temporal population dynamics. In: Marret, F., Lewis, J. M. \& Bradley, L. R. (Eds.). Biological and geological perspectives of dinoflagellates (pp 149-153). London: Geological Society of London

EPA (2015). Connectivity of streams and wetlands to downstream waters: A review and synthesis of the scientific evidence. Environmental Protection Agency 600/R-14/475F, January 2015. https://cfpub.epa.gov/ncea/risk/recordisplay. cfm?deid=296414

Finlay, B.J. \& Esteban, G. F. (1998). Planktonic ciliate species diversity as an integral component of ecosystem function in a freshwater pond. Protist, 149, 155-165 https://doi.org/10.1016/ S1434-4610(98)70020-3

Foissner, W., Berger, H. \& Schaumburg, J. (1999). Identification and ecology of limnetic plankton ciliates. Munich: Bavarian State Office for Water Management.

Gannon, J. E. (1971). Two counting cells for the enumeration of zooplankton micro-crustacea.
Transactions of the American Microscopical Society, 4, 486-490. DOI: 10.2307/3225467

García, M. (2011). El cinturón hortícola platense: ahogándonos en un mar de plásticos. Un ensayo acerca de la tecnología, el ambiente y la política. Tehomai, 23, 35-53.

Gómez, N. \& Licursi, M. (2001). The Pampean Diatom Index (IDP) for assessment of rivers and streams in Argentina. Aquatic Ecology, 35, 173 181. https://doi.org/10.1023/A:1011415209445.

Gómez, N., Licursi, M. \& Cochero, J. (2009). Seasonal and spatial distribution of the microbenthic communities of the Río de la Plata estuary (Argentina) and possible environmental controls. Marine Pollution Bulletin, 58, 878-887. https://doi.org/10.1016/j. marpolbul.2009.01.014.

Haberman, J. \& Haldna, M. (2014). Indices of zooplankton community as valuable tools in assessing the trophic state and water quality of eutrophic lakes: long term study of Lake Võrtsjärv. Journal of Limnology, 73(2), 263-273. DOI: 10.4081/jlimnol.2014.828

Hairston Jr, N. G. (1996). Zooplankton egg banks as biotic reservoirs in changing environments. Limnology and Oceanography, 41(5), 1087-1092. https://doi.org/10.4319/lo.1996.41.5.1087

Hindák, F., Wolowski, K. \& Hindáková, A. (2000). Cysts and their formation in some neustonic Euglena species. Annales de LimnologieInternational Journal of Limnology, 36(2), 83-93. https://doi.org/10.1051/limn/2000010

Jeppesen, E., Kristensen, P., Jensen, J. P., Søndergaard, M., Mortensen, E. \& Lauridsen, T. (1991). Recovery resilience following a reduction in external phosphorus loading of shallow, eutrophic Danish lakes: duration, regulating factors and methods for overcoming resilience. Memorie dell'Istituto Italiano di Idrobiologia, 48(1), 127-148.

Jeppesen, E., Nöges, P., Davidson, T. A., Haberman, J., Nöges, T., Blank, K., Lauridsen, T., et al. (2011). Zooplankton as indicators in lakes: a scientific-based plea for including zooplankton in the ecological quality assessment of lakes according to the European Water Framework 
Directive (WFD). Hydrobiologia, 676, 279-297. https://doi.org/10.1007/s10750-011-0831-0

Junk, W. J., Bayley, P. B. \& Sparks, R. E. (1989). The flood pulse concept in river-floodplain systems. Canadian Special Publication of Fisheries and Aquatic Sciences, 106(1), 110-127.

Komárek, J. \& Fott, B. (1983). Das Phytoplankton des Süsswassers 7 (1) Chlorophyceae: Chlorococcales. Stuttgart: E. Schweizerbart'sche Verlagsbuchhandlung (Nagele u. Obermiller).

Komárek, J. \& Anagnostidis, K. (2005). Süsswasserflora von Mitteleuropa 19 (2), Cyanoprokaryota: Oscillatoriales. Italy: Elsevier, Spectrum Akademischer Verlag.

Koste, W. (1978). Rotatoria. Die Radertiere Mitteleuropas. Uberordnung Monogononta. Stuttgart: Gebrüder Borntraeger.

Krammer, K. \& Lange-Bertalot, H. (1986). Bacillariophyceae. 1. Teil: Naviculaceae. In: Ettl, H., Gerloff, J., Heynig, H. \& Mollenhauer, D. (Eds). Süsswasser flora von Mitteleuropa, 2/1 (pp 1-876). Stuttgart, New York: Gustav Fischer Verlag

Krammer, K. \& Lange-Bertalot, H. (1988). Bacillariophyceae. 2. Teil: Bacillariaceae, Epithemiaceae, Surirellaceae. In: Ettl, H., Gerloff, J., Heynig, H. \& Mollenhauer, D. (Eds). Süsswasserflora von Mitteleuropa, 2/2 (pp. 1-596). Jena: VEB Gustav Fischer Verlag.

Krammer, K. \& Lange-Bertalot, H. (1991a). Bacillariophyceae. 3. Teil: Centrales, Fragilariaceae, Eunotiaceae. In: Ettl, H., Gerloff, J., Heynig, H. \& Mollenhauer, D. (Eds). Süsswasserflora von Mitteleuropa, 2/3 (pp 1-576). Stuttgart, Jena: Gustav Fischer Verlag.

Krammer, K. \& Lange-Bertalot, H. (1991b). BacillariophyceaeTeil 4: Achnanthaceae, Literaturverzeichnis. In: Ettl, H., Gerloff, J., Heynig, H. \& Mollenhauer, D. (Eds). Süsswasserflora von Mitteleuropa, Vol 2. Stuttgart: Gustav Fischer Verlag.

Lange-Bertalot, H. (2000). Iconographia Diatomologica - Annotated Diatom Micro-graphs. Vol. 9: Diatoms of the Andes, from Venezuela to Patagonia/Tierra del Fuego. Ruggell: A. R. G. Gantner Verlag.
Lee, A. A. \& Bukaveckas, P. A. (2002). Surface water nutrient concentrations and litter decomposition rates in wetlands impacted by agriculture and mining activities. Aquatic Botany, 74(4), 273-285.

Licursi, M. \& Gómez, N. (2002). Benthic diatoms and some environmental conditions in three lowland streams. International Journal of Limnology - Annales de Limnologie, 38, 109-118. https://doi.org/10.1051/limn/2002009.

López, I. (2018). Inundaciones por la lluvia en el sur de la Región Metropolitana de Buenos Aires: riesgosy estrategia para la acción. Ciudad Autónoma de Buenos Aires: Espacio Editorial.

Lund, J. W. G., Kipling, C. \& Le Cren, E. D. (1958). The inverted microscope method of estimating algal numbers and the statistical basis of estimations by counting. Hydrobiologia, 11, 143-170. https:// doi.org/10.1007/BF00007865

Mac Loughlin, T. M., Peluso, L. \& Marino, D. J. (2017). Pesticide impact study in the periurban horticultural area of Gran La Plata, Argentina. Science of the Total Environment, 598, 572-580. https://doi.org/10.1016/j.scitotenv.2017.04.116

McQuoid, M., Godhe, A. \& Nordberg, K. (2002). Viability of phytoplankton resting stages in the sediments of a coastal Swedish fjord. European Journal of Phycology, 37(2), 191-201. https://doi. org/10.1017/S0967026202003670

Medley, K. A. \& Havel, J. E. (2007). Hydrology and local environmental factors influencing zooplankton communities in floodplain ponds. Wetlands, 27(4), 864-872.

Metzeltin, D. \& Lange-Bertalot, H. (1998). Tropical diatoms of South America I. About 700 predominantly rarely known or new taxa representative of the neotropical flora. LangeBertalot, H. (Ed.). Iconographia Diatomologica, 5, 1-695.

Metzeltin, D. \& Lange-Bertalot, H. (2005). Diatoms of Uruguay. Compared with other taxa from South America and elsewhere. Lange-Bertalot, H. (Eds). Iconografia Diatomologica, 15, 1-736.

Metzeltin D. \& Lange-Bertalot, H. (2007). Tropical Diatoms of South America II. Special remarks 
on biogeographic disjunction. Lange-Bertalot, H. (Eds). Iconografia Diatomologica, 18, 1-877.

Meyer. J. J. (1994). The microbial loop in flowing waters. Microbiol Ecology, 28, 195-199. https:// doi.org/10.1007/BF00166808

Millennium Ecosystem Assessment (2005). Ecosystems and human well-being: synthesis. Washington, DC: Island Press.

Mitsch, W. J. \& Wilson, R. F. (1996). Improving the success of wetland creation and restoration with know how, time, and self design. Ecological applications, 6(1), 77-83. https://doi. org/10.2307/2269554

Modenutti, B. E. (1991). Zooplancton de ambientes lóticos de la subcuenca Delta del río Paraná, Buenos Aires, Argentina. Iheringia (Série Zoologia), 71, 67-80.

Nogrady, T. \& Segers, H. (2005). Guides to the identification of the microinvertebrates of the continental waters of the world. Dumont H.J.F (Coordinating editor). The Netherlands: Backhuys Publishers.

Prygiel, J. \& Leitao, M. (1994). Cyanophycean blooms in the reservoir of Val Joly (northern France) and their development in downstream rivers. In: Descy, J. P., Reynolds, C. S.,\& Padisák J. (Eds). Phytoplankton in Turbid Environments: Rivers and Shallow Lakes (pp. 85-96). Dordrecht: Springer. https://doi.org/10.1007/978-94-0172670-2_8

Reid, J. W. (1985). Chave de identificação e lista de referências bibliográficas para as espécies continentais sulamericanas de vida livre da ordem Cyclopoida (Crustacea, Copepoda). Boletim de Zoologia Universidade São Paulo, 9, 17-143.

Rimoldi, F., Peluso, L., Rossini, G. B., Ronco, A. E. \& Demetrio, P. M. (2018). Multidisciplinary approach to a study of water and bottom sediment quality of streams associated with mixed land uses: Case study Del Gato Stream, La Plata (Argentina). Ecological indicators, 89, 188-198. https://doi.org/10.1016/j. ecolind.2018.01.063

Ringuelet, R. A. (1958). Los crustáceos copépodos de las aguas continentales de la República
Argentina. Sinópsis sistemática. Serie Zoología, 1(2), 35-125.

Ringuelet, R. A. (1962). Ecología acuática continental. Buenos Aires: EUDEBA.

Rodrigues Capítulo, L., Kruse, E., y Gómez, N. (2020). Los bañados de desborde fluvial: una mirada desde la geohidrología. Biología Acuática, 35.

Rodrigues, L. C., Simões, N. R., Bovo-Scomparin, V. M., Jati, S., Santana, N. F., Roberto, M. C., et al. (2015). Phytoplankton alpha diversity as an indicator of environmental changes in a neotropical floodplain. Ecological Indicators, 48, 334-341. https://doi.org/10.1016/j. ecolind.2014.08.009

Romaní, A. M., Artigas, J., Camacho, A., GraÇa, M. A. \& Pascoal, C. (2009). La biota de los ríos: los microorganismos heterotróficos. En: Elosegi, A. \& Sabater, S. (Eds). Conceptos y técnicas en ecología fluvial (pp. 169-218). Bilbao: Fundación BBVA.

Round, F. E. (1990). The effect of liming on the benthic diatom populations in three upland welsh lakes. Diatom Research, 5, 129-140. https:// doi.org/10.1080/0269249X.1990.9705098

Sanders, P. F. \& Webster, J. (1978). Survival of aquatic hyphomycetes in terrestrial situations. Transactions of the British Mycological Society, 71(2), 231-237. https://doi.org/10.1016/S00071536(78)80103-X

Sati, S. \& Belwal, M. (2005). Aquatic hyphomycetes as endophytes of riparian plant roots. Mycologia, 97, 45-49. https://doi.org/10.1080/15572536.20 06.11832837

Schwarz, M. V. J. \& Frenzel, P. (2003). Population dynamics and ecology of ciliates (Protozoa, Ciliophora) in an anoxic rice field soil. Biology and Fertility of Soils, 38, 245-252. https://doi. org/10.1007/s00374-003-0644-z

Sigee, D.C. (2019). Microorganisms 1. Phytoplankton, attached algae, and biofilms. In: Hughes J. M. R. (Ed.). Freshwater ecology and conservation: Approaches and techniques (pp. 133). UK: Oxford University Press. doi:10.1093/ oso/9780198766384.003.0007 
Souffreau, C., Vanormelingen, P., Sabbe, K. \& Vyverman, W. (2013). Tolerance of resting cells of freshwater and terrestrial benthic diatoms to experimental desiccation and freezing is habitat-dependent. Phycologia, 52(3), 246-255. https://doi.org/10.2216/12-087.1

Seifert, K. A. \& Gams, W. (2011). The genera of Hyphomycetes. Persoonia Molecular Phylogeny and Evolution of Fungi, 27, 119-129. https://doi. org/10.3767/003158511X617435

Solari, L. C., Quaíni, K. P. \& Gabellone, N. A. (2018). Succession of microconsumers in waterlogged pampean soils (Buenos Aires, Argentina) and its significance for nearby wetlands. Aquatic Sciences, 80, 42. doi.org/10.1007/s00027-0180593-0

Solé, M., Fetzer, I., Wennrich, R., Sridhar, K. R., Harms, H. \& Krauss, G. (2008). Aquatic hyphomycete communities as potential bioindicators for assessing anthropogenic stress. Science of the total environment, 389(2-3), 557-565. https://doi.org/10.1016/j. scitotenv.2007.09.010

Solís, M., Bonetto, C., Marrochi, N., Paracampo, A. \& Mugni, H. (2018). Aquatic macroinvertebrate assemblages are affected by insecticide applications on the Argentine Pampas. Ecotoxicology and Environmental Safety, 148, 11-16. https://doi.org/10.1016/j. ecoenv.2017.10.017

Spencer, M. \& Blaustein, L. (2001). Risk of predation and hatching of resting eggs in the ostracod Heterocypris incongruens. Journal of Crustacean Biology, 21, 575-581. https://doi. org/10.1163/20021975-99990159

Stevenson, B. R. J. \& Bahls, L. L. (1999). Periphyton protocols. In: Barbour, M. T., Gerritsen, J., Snyder, B. D \& Stribling, J. B. (Eds.). Rapid bioassessment protocols for use in wadeable streams and rivers: Periphyton, benthic macroinvertebrates and fish. (Second Edition) (pp. 23). Washington: EPA.

Stockner, J. G. \& Lund, J. W. G. (1970). Live algae in postglacial lake deposits. Limnology and Oceanography, 15, 41-58. https://doi. org/10.4319/lo.1970.15.1.0041
Strayer, D. L., \& Dudgeon, D. (2010). Freshwater biodiversity conservation: recent progress and future challenges. Journal of the North American Benthological Society, 29(1), 344-358 https://doi. org/10.1899/08-171.1

Tarda, A. S., Saparrat, M. C. N. \& Gómez, N. (2019). Assemblage of dematiaceous and Ingoldian fungi associated with leaf litter of decomposing Typha latifolia L. (Typhaceae) in riverine wetlands of the Pampean plain (Argentina) exposed to different water quality. Journal of Environmental Management, 250, 109409. https://doi.org/10.1016/j.jenvman.2019.109409

Tell, G. \& Conforti, V. (1986). Euglenophyta Pigmentadas de la Argentina. Bibliotheca Phycologica, Band 75. Berlin-Stuttgart: J. Cramer.

Tudor, M., Tudor I.-M., Ibram, O., Teodorof, L., Nastase, C. \& Deak, G. (2015). Analysis of biological indicators related to the surface water quality in Danube Delta Biosphere Reserve. Journal of Environmental Protection and Ecology, 16 (2), 443-452.

Utermöhl, H. (1958). Zur Vervollkommnung der quantitativen Phytoplankton-Methodik. Internationale Vereinigung für Theoretische und Angewandte Limnologie: Mitteilungen, 9, 1-38. https://doi.org/10.1080/05384680.1958.119 04091

Vijverberg, J. (1977). Population structure, life histories and abundance of copepods in Tjeukemeer, The Netherlands. Freshwater Biology, 7(6), 579-597. https://doi. org/10.1111/j.1365-2427.1977.tb01710.x

Zaplara, V. S., Solari, L. C., Benítez, H. H. \& Gabellone, N. A. (2018). Microorganismos consumidores en suelos de la llanura de inundación del arroyo El Pescado (Buenos Aires): Experiencia en microcosmos. Revista del Museo Argentino Ciencias Naturales nueva serie, 20(2): 311-322. http://hdl.handle.net/11336/85448

Zuur, A. F., leno, E. N. \& Elphick, C. S. (2010). A protocol for data exploration to avoid common statistical problems. Methods in Ecology and Evolution, 1, 3-14. https://doi.org/10.1111/ j.2041-210X.2009.00001.x 